\title{
Études archéologiques d'enduits peints : les exemples des chantiers de Cergy, Souvigny et Soissons
}

\section{Emmanuelle Boissard-Stankov}

\section{(2) OpenEdition \\ 1 Journals \\ Édition électronique \\ URL : https://journals.openedition.org/cem/897 \\ DOI : $10.4000 /$ cem. 897 \\ ISSN : 1954-3093 \\ Éditeur \\ Centre d'études médiévales Saint-Germain d'Auxerre}

\section{Édition imprimée}

Date de publication : 15 août 2004

ISSN : 1623-5770

Référence électronique

Emmanuelle Boissard-Stankov, «Études archéologiques d'enduits peints : les exemples des chantiers de Cergy, Souvigny et Soissons », Bulletin du centre d'études médiévales d'Auxerre I BUCEMA [En ligne], 8| 2004, mis en ligne le 13 décembre 2006, consulté le 22 septembre 2022. URL : http:// journals.openedition.org/cem/897 ; DOI : https://doi.org/10.4000/cem.897

Ce document a été généré automatiquement le 22 septembre 2022.

\section{(c) (i) (2) (2)}

Creative Commons - Attribution - Pas d'Utilisation Commerciale - Partage dans les Mêmes Conditions 4.0 International - CC BY-NC-SA 4.0

https://creativecommons.org/licenses/by-nc-sa/4.0/ 


\title{
Études archéologiques d'enduits peints : les exemples des chantiers de Cergy, Souvigny et Soissons
}

\author{
Emmanuelle Boissard-Stankov
}

1 Les trois chantiers présentés illustrent l'étendue du champ de recherche archéologique sur les enduits peints : de l'étude stratigraphique d'enduits conservés in situ (Cergy) ou de l'analyse macroscopique de fragments trouvés en fouille (Souvigny), à l'identification physico-chimique des pigments (Soissons).

Cergy-Pontoise (Val d'Oise), l'église Saint-Christophe

2 Pour parvenir à la restitution chronologique des états successifs du décor mural ${ }^{1}$, les résultats de l'observation systématique des différentes couches d'enduit conservées dans l'ensemble de l'édifice sont confrontés aux données archéologiques et historiques connues pour le site. Une analyse stylistique et comparative des décors offre des indications supplémentaires pour une datation.

3 L'étude stratigraphique détaillée des enduits peints de l'église Saint-Christophe de Cergy sera intégrée au rapport archéologique des fouilles réalisées sous la responsabilité de Samuel Riou. Quatre états de décor peuvent être attribués à l'époque médiévale :

4 - au XII ${ }^{\mathrm{e}}$ siècle, l'appareil de calcaire fin présente un parement au layage et aux joints minces dont le réseau est souligné d'un enduit beurré, lissé et incisé. L'ensemble adopte un aspect particulièrement soigné, qui pourrait avoir subsisté en l'état jusqu'à l'agrandissement du chevet au XIII ${ }^{e}$ siècle ;

5 - à la suite de ces travaux, un enduit lissé et de teinte proche de la pierre - brun clair ponctuellement plus proche du rose ou du jaune - recouvre l'ensemble des élévations, et porte un décor de faux-joints blancs ;

6 - à ces élévations homogènes, au décor structurel et quasi monochrome, se substitue un espace architectural vivement coloré dissociant parties basses et couvrement. Les murs sont peints d'un jaune soutenu et lumineux ponctuellement rehaussé de rouge et de faux- 
joints blancs, alors que le décor précédent de faux-joints blancs sur fond brun clair subsiste dans les parties hautes et les voûtes;

7 - un nouveau décor de faux-joints blancs sur fond d'enduit gris recouvre l'ensemble des élévations à la fin de l'époque médiévale, et constitue la première couche observée sur l'un des supports de la chapelle nord, datée du XIV siècle.

8 Deux motifs identifiés comme des croix de consécration apparaissent successivement en deux endroits de l'édifice. Les plus anciennes se situent stratigraphiquement sur le décor coloré jaune vif. Les plus récentes ont été peintes sur l'enduit gris à faux-joints blancs, et pourraient correspondre à la reconsécration de l'église en 1479, mentionnée par l'abbé Bourguignon dans une notice historique datée du 27 juillet 1870.

Souvigny (Allier), le tombeau des saints abbés Maïeul et Odilon de Cluny

9 Près de 1500 fragments d'enduit peint, découverts lors de la fouille du tombeau des saints abbés de Cluny ${ }^{2}$, ont fait l'objet d'une étude macroscopique, première étape nécessaire à toute analyse de ce type de mobilier en grande quantité. Deux fiches descriptives exploitables sous le logiciel de base de données File Maker Pro ont été établies ; la première recueille les caractères généraux de mise en œuvre des supports (stratigraphie, type d'enduits, de badigeons et de couches picturales composant les fragments), la seconde permet une description détaillée du décor (stratigraphie, couleurs, type de motif...) ${ }^{3}$. L'aspect systématique de ce type d'étude a pour premier résultat de faciliter l'assemblage des fragments, et donc la reconstitution de motifs. L'identification des types de support contribue également au rapprochement de fragments dont les motifs n'appartiennent a priori pas au même registre, et donc à la restitution d'un programme décoratif. La prise en compte des différentes couches stratigraphiques et leur caractérisation permettent de formuler des hypothèses techniques sur la mise en œuvre des enduits et couches picturales (plusieurs états de décor, préparation particulière à son application, richesse de la palette de pigments...).

10 À Souvigny, les fragments appartiennent au décor d'un premier monument funéraire élevé autour du sarcophage à la fin du $\mathrm{XI}^{\mathrm{e}}$ siècle et réaménagé lors de l'installation d'un ciborium en bois. Ils se situent dans une étroite fourchette chronologique comprise entre la construction de ce premier monument et sa destruction après un incendie au milieu du $\mathrm{XII}$ s.

11 L'utilisation d'un sable quartzeux, probablement celui de l'Allier, est commune à tous les types décrits. Chaque support est constitué d'une à quatre couches distinctes, d'enduit gris blanc plus ou moins riche en charge de granulométrie variable, ou d'enduit rose de tuileau finement broyé mêlé à du sable de granulométrie grossière. Le mélange est hétérogène : à la charge minérale s'ajoutent des charbons de bois et des nodules de chaux dans la majorité des enduits, des nodules terreux et des fibres végétales dans les couches inférieures les plus grossières.

12 Sur les quatre groupes de fragments distingués, deux présentent deux états de décor qui pourraient correspondre aux deux phases de construction du monument funéraire. Les motifs sont d'inspiration géométrique ou imitent des placages de marbre moucheté. Dans la seconde phase, des tracés blancs sur fond vert pourraient appartenir à une inscription.

13 La couche picturale n'a pas été appliquée sur un badigeon mais directement sur l'enduit, probablement encore frais, excepté le pigment noir, très pulvérulent. 

profond et au blanc utilisé en rehaut s'ajoutent du vert, du bleu, du jaune, du rouge, de l'orange et du mauve. La variété de cette palette accroît la diversité des gammes de polychromie et ajoute à la richesse recherchée dans l'imitation des placages de marbres mouchetés.

Soissons (Aisne), l'abbaye Saint-Jean-des-Vignes

15 de 3000 fragments d'enduits peints, mis au jour entre 1983 et 2000. Ces fragments ont fait l'objet d'une analyse archéologique macroscopique en $2002{ }^{4}$. En accord avec les données archéologiques de terrain recueillies par Sheila Bonde (Brown University USA) et Clark Maines (Wesleyan University - USA) ${ }^{5}$, les résultats ont permis de relier certains types de décor à des espaces spécifiques de l'abbaye : sacristie, salle capitulaire, cloître, réfectoire... Le répertoire décoratif, typiquement roman, confirme la datation donnée par la position stratigraphique des fragments, entre la fondation de l'abbaye romane en 1076 et un terminus post quem aux environs de 1230, date de la reconstruction gothique. La reconstitution des motifs donne une première image de l'ornementation des bâtiments conventuels d'une abbaye romane en France septentrionale.

16 À ce stade, il a paru intéressant de poursuivre les recherches sur les enduits peints de l'abbaye Saint-Jean-des-Vignes de Soissons, en complétant l'étude macroscopique déjà effectuée par une analyse des pigments.

17 La méthode choisie a été la spectrométrie Raman. Cette méthode repose sur le principe de l'interaction entre le rayonnement et la matière : l'échantillon est soumis à un rayonnement électromagnétique (le faisceau monochromatique du laser) et le spectre de vibration Raman obtenu est caractéristique de la molécule en présence. Cette méthode présente plusieurs avantages :

18 - elle ne nécessite aucun prélèvement ni aucune préparation spécifique, ce qui garantit la stricte conservation des enduits peints ;

19 - en microscopie confocale, elle permet l'analyse des couches inférieures d'un matériau stratifié, en conservant ses propriétés non destructives.

20 Les analyses ont été effectuées au Laboratoire de Dynamique, Interactions et Réactivité (LADIR) de Thiais (Val-de-Marne), sous la responsabilité de Claude Coupry, ingénieur de recherche au CNRS.

21 Les résultats détaillés de cette étude seront présentés dans une publication sur l'ensemble du site de l'abbaye Saint-Jean-des-Vignes de Soissons, à paraître sous la direction de $\mathrm{C}$. Maines et $\mathrm{S}$. Bonde. Les différences techniques observées à l'échelle macroscopique ont trouvé un écho dans la diversité des pigments utilisés, et permettent d'en déduire de nouvelles hypothèses concernant la chronologie relative. Une partie des matières colorantes utilisées, ocres jaune et rouge et terres vertes, correspond à une palette "commune" dans ce contexte régional roman, probablement d'origine locale. D'autres dénotent une recherche particulière, et induisent la participation à un réseau commercial de plus grande ampleur.

L'étude de ces enduits serait complète avec une observation des stratigraphies en microscopie optique sur section polie et une analyse pétrographique sur lame mince. Ce type d'analyse comporte l'inconvénient d'être destructeur, mais l'étude macroscopique préalablement réalisée permet la sélection de quelques échantillons représentatifs des

Bulletin du centre d'études médiévales d'Auxerre | BUCEMA, 8 | 2004 
types de support et de décor. Une collaboration, semblable à celle qui a pu s'initier avec le LADIR, pourrait s'instaurer avec un laboratoire intéressé par ce type de travaux.

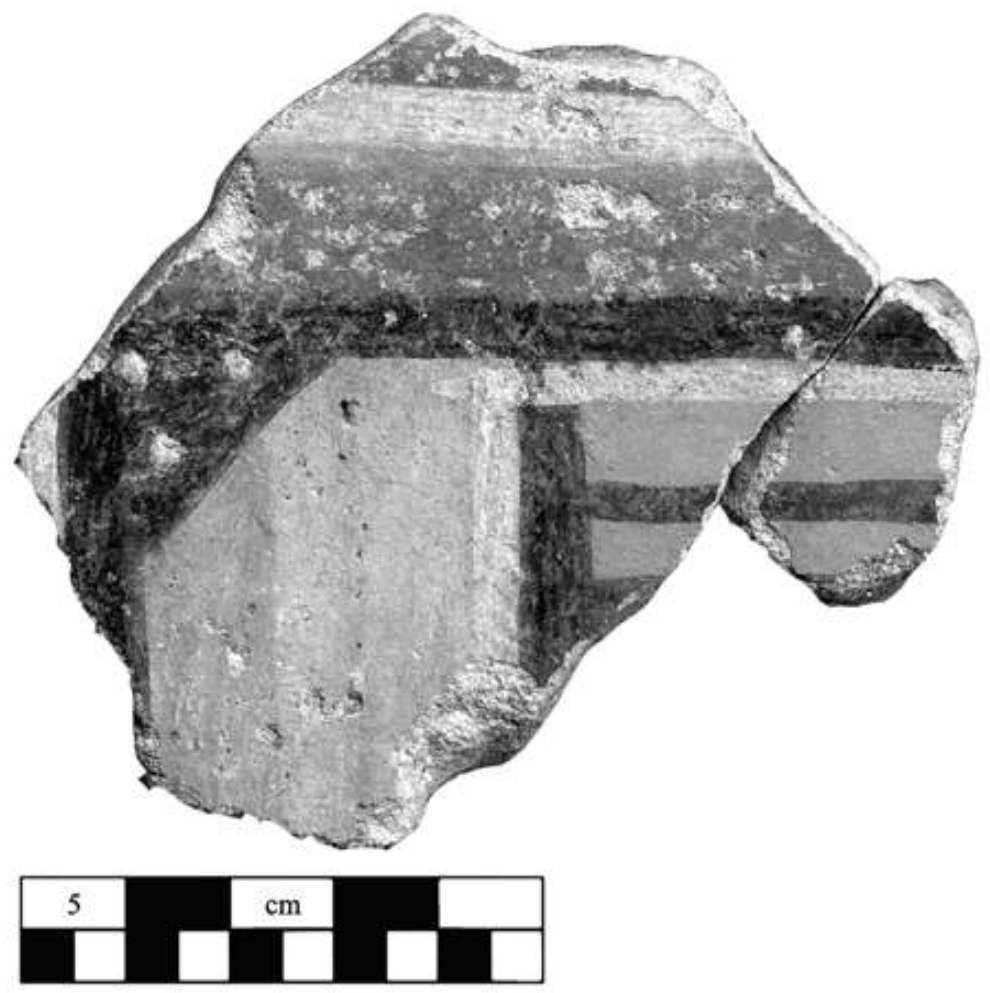

Soissons, abbaye Saint-Jean-des-Vignes. Fragment d'enduit peint (E. Boissard-Stankov).

\section{NOTES}

1.. C. SAPIN, E. BOISSARD, Le Villars (Saône-et-Loire). Analyse et relevés archéologiques, Rapport, Auxerre, octobre 2001 ; E. BOISSARD, L'église Saint-Vincent de Sologny : étude du bâti et des enduits, Lyon, Université Lumière Lyon 2, maîtrise d'histoire de l'art et archéologie, sous la dir. J.-F. REYNAUD, 1999, 3 vol.

2.. P. CHEVAlIER, A. MAQUET, Des tombeaux, des reliques et des saints. Découverte des tombeaux et gisants des saints abbés de Cluny, Catalogue d'exposition, Musées de Souvigny, 2003. 3.. E. BOISSARD-STANKOV, Abbaye Saint-Jean-des-Vignes (Aisne). Analyse d'un ensemble d'enduits peints, Rapport, SRA Picardie-Amiens, Auxerre, août 2002.

4.. Ibid.

5.. S. BONDE, C. MAINES (éd.), Saint-Jean-des-Vignes in Soissons. Approaches to its architectures, archaelogy and history, Brepols, Turnhout, 2003 (Bibliotheca Victorina XV). 
INDEX

Index géographique : France/Cergy-Pontoise, France/Soissons, France/Souvigny

Mots-clés : enduit peint 